\title{
SEVEN YEAR FOLLOW-UP OF AGE-RELATED MACULOPATHY IN AN ELDERLY BRITISH POPULATION
}

\author{
J. M. SPARROW 1 , A. J. DICKINSON ${ }^{2}$, A. M. DUKE ${ }^{1}$, J. R. THOMPSON ${ }^{3}$ J. M. GIBSON ${ }^{4}$ and \\ A. R. ROSENTHAL ${ }^{3}$ \\ Bristol, Newcastle upon Tyne, Leicester and Birmingham
}

\begin{abstract}
SUMMARY
Despite age-related macular degeneration (AMD) being the commonest cause of blindness amongst the elderly in Western society, the incidence of new lesions is poorly documented and the natural history of existing disease remains ill understood.

Purpose: To document in an elderly population the incidence of new AMD lesions and the progression of pre-existing AMD over time.

Method: Baseline ophthalmic examinations were performed on a geographically defined random population sample of elderly people in 1982-4, and retinal photographs taken. The present study re-examined and re-photographed survivors after approximately 7 years using the same fundus camera. Photographs were randomly encoded, and independently graded for AMD features by two masked observers using the Wisconsin AMD grading system. Disagreements were resolved by review to reach a consensus.

Results: Eighty-two of the 88 participating survivors had photographs of gradable quality on both occasions in at least one eye. Mean age at follow-up was 87 years (range 84-97 years) and 70.7\% of subjects were female. Paired photographs were available on 158 eyes, and showed important differences in drusen type, drusen area and characteristics of the retinal pigment epithelium (RPE) between initial and subsequent examinations. The 7 year incidence (and regression) of lesions was: drusen $30.6 \%(20.0 \%)$, RPE degeneration $54.5 \%$ $(8.8 \%)$, increased pigment $11.6 \%(64.7 \%)$, subretinal baemorrhage $1.3 \%$, subretinal scar/fibrin $1.3 \%$ and geographic study $1.3 \%$.
\end{abstract}

From: ${ }^{1}$ University Department of Ophthalmology, Bristol Eye Hospital, Bristol; ${ }^{2}$ Department of Ophthalmology, Royal Victoria Infirmary, Newcastle upon Tyne; ${ }^{3}$ University Department of Ophthalmology, Leicester Royal Infirmary, Leicester, ${ }^{4}$ Department of Ophthalmology, Birmingham Heartlands Hospital, Birmingham, UK.

Correspondence to: $\mathrm{Mr}$ J. M. Sparrow, DPhil, FRCS, FRCOphth, University Department of Ophthalmology, Bristol Eye Hospital, Bristol BS1 2LX, UK. Fax: +44 (117) 9251421.
Conclusion: These unique population-based results provide new insight into the natural history of AMD in an elderly population.

In Western society it is well recognised that the commonest cause of blindness in the elderly is agerelated macular degeneration (AMD). ${ }^{1-3}$ To date, however, the natural history of this disease has been poorly documented. ${ }^{4}$ Representative populationbased incidence and natural history data are not available for AMD - a fact which precludes rational planning for the health care needs of a growing population of elderly people who are at risk of visual impairment from AMD. A single published cohort study of men involved in just one occupation has provided 5 year incidence figures for certain AMD features, although this population was relatively young, with almost half the participants being under 50 years of age and only $11 \%$ over 70 years at baseline. ${ }^{5}$ Hospital-based natural history studies have generally focused on exudative AMD. ${ }^{6,7}$ Whilst accounting for much of the legal blindness associated with AMD, this represents only a small proportion of the spectrum of AMD and its attendant morbidity. ${ }^{8}$ Without detailed basic data on all forms of AMD, understanding of this condition remains limited.

Detailed documentation of AMD requires a comprehensive grading system with acceptable repeatability, one example of which is the Wisconsin Age-related Maculopathy Grading System. ${ }^{9,10}$ Many authors define AMD as the presence of specified lesions only in the context of visual impairment. ${ }^{11-13}$ For natural history studies, however, this definition is inappropriate, because AMD lesions associated with normal vision at baseline may show visual loss over time, which may or may not be due to AMD. In addition, omitting description of those eyes with AMD lesions and normal vision would disregard vast 
amounts of data which are fundamental to a better understanding of the natural history of this disease. ${ }^{4}$

The purpose of this study was to examine the natural history of AMD in a geographically defined random population sample of elderly people (77 years and over). Using fundus photographs, the incidence of new lesions and the progression of existing lesions over a 7 year period was observed.

\section{METHOD}

Elderly residents of the English town of Melton Mowbray, Leicestershire, were studied. In terms of the age and sex distributions, social class, and urban or rural residence, the population of this town is highly representative of England and Wales as a whole ${ }^{14}$ which makes it a particularly valuable epidemiological resource. As described in detail elsewhere,$^{15}$ the survivors of a population-based random sample of elderly individuals formed the target population of the present follow-up study. Baseline ophthalmic assessment took place between 1982 and 1984 and included a complete ophthalmic assessment and fundus photography $\left(30^{\circ}\right.$ macular colour transparencies) using a non-stereoscopic Zeiss Jena fundus camera. ${ }^{16}$ Survivors of the earlier eye study were re-identified after 7 years, and invited to participate in a follow-up ophthalmic examination which took place in 1990. The participating survivors were re-examined and re-photographed using the same fundus camera. ${ }^{15}$

The fundus photographs (mounted colour transparencies) from initial and subsequent assessments were randomly encoded and a grid defining nine subfields superimposed over the macula according to the protocol of the Wisconsin Age-related Maculopathy Grading System. ${ }^{9}$ In this system the macula is subdivided by three concentric circles and four diagonals into a grid containing one central, four inner and four outer subfields. Features which were graded included maximum drusen size and predominant (most common) drusen size, predominant drusen type (those occupying largest area), drusen area and confluence of drusen, retinal pigment epithelial (RPE) degeneration, increased pigmentation, subretinal haemorrhage, subretinal scar and/or fibrin deposition, and geographic atrophy of the macula. Predominant drusen size and drusen con-

fluence were graded for the macula as a whole (by eye), all other features being graded for each of the nine retinal subfields individually. A number of minor modifications were required to facilitate the grading of non-stereoscopic images. ${ }^{10,15}$ Retinal images were graded independently by two masked observers (A.J.D. and J.M.S.), with grading disagreements being resolved by review to reach a consensus. Inter-observer variability between the two graders was assessed prior to unmasking by weighted and unweighted kappa statistics and by percentage agreements. The levels of agreement were generally moderate to substantial. ${ }^{10}$

We report the percentage of eyes initially free of each feature which went on to develop that feature during the period of follow-up. We refer to this measure as the 7 year incidence. Changes in disease status over the 7 year period were assessed in two ways, and were expressed in a 'by eye' format.

Firstly, in this study the incidence of new lesions over time was defined as the percentage of eyes free of a particular feature at initial assessment which subsequently developed that feature at the follow-up assessment. Although many lesions in the subjects studied will have developed well before the time of follow-up, and some of the subjects who developed lesions will have died before follow-up, the term incidence (as defined above) has been used in the interests of simplicity. In general, the 'diagnostic threshold' for the development of a particular feature corresponded to progression from 'absent' or 'questionable' grades to feature 'definitely present'. In addition to the incidence rate over the 7 year period, an equivalent regression rate was determined. Because of the slightly insecure assumption underlying the rank-ordering of drusen type; i.e. hard, soft and reticular, multiple diagnostic thresholds were used for this feature. These were based strictly on presence or absence of items at a particular level on the scale. Occasionally an additional and more detailed focus on local retinal changes within a subfield or defined group of subfields was considered appropriate, and these are presented to highlight particular points of interest. The incidence/regression data were formatted as tables.

Secondly, a statement regarding the progression of existing disease was produced. This was defined simply as the arithmetic change in grade over the 7 year period. For a given eye, progression of a particular feature would provide a positive value, whilst regression would provide a negative value, with unchanged features scoring zero. These results were formatted as frequency distributions for the change in score. Where features were either absent or doubtfully present on both occasions this was separately indicated.

In the Wisconsin system the 'predominant drusen type' for each individual subfield is assessed? This results in difficulty when attempting to describe the predominant drusen type for an eye as a whole because the areas of the subfields vary. In the absence of stereo images a size criterion was used as one of the diagnostic determinants of drusen type. $^{10,15}$ This slight departure from the Wisconsin protocol offered the opportunity of defining a 'proxy measure' of drusen type using the 'predominant drusen size' variable. This variable had the advan- 
tage of referring, from the outset, to the eye as a whole. In this paper drusen type for the eye as a whole is thus presented in two slightly different and complementary ways. The first represents the highest scoring subfield from a subfield-by-subfield assessment of 'predominant drusen type', and the second represents a 'proxy measure' based on the 'predominant drusen size' for the eye as a whole.

The complexity of the Wisconsin system encouraged us to define a number of 'clinically relevant' summary measures based on the detailed grading. Thus AMD status was summarised by classifying eyes into three categories:

1. None: AMD features scored as absent or questionable in the worst affected subfield. (Indistinct small hard drusen were included in this category.)

2. Minor $A M D$ : drusen which were not reticular, with confluence $<500 \mu \mathrm{m}$, and with total drusen area occupying $<10 \%$ of the total grid; RPE degeneration occupying $<25 \%$ of the grid; increased pigmentation.

3. Major $A M D$ : reticular drusen, drusen with confluence $\geqslant 500 \mu \mathrm{m}$, drusen occupying $\geqslant 10 \%$ of the total grid area; RPE degeneration involving $\geqslant 25 \%$ of the total grid area; exudative AMD (subretinal haemorrhage or scar/fibrin); geographic atrophy.

The study was approved by the ethics committee with responsibility for research in the Leicester group of hospitals and all subjects gave informed verbal consent prior to participation.

\section{RESULTS}

The target population of the present study consisted of the survivors of the 529 participants of an earlier eye study. ${ }^{16}$ The details of the present study population have been described elsewhere. ${ }^{15}$ Briefly, in 1990 the known survivors (survivorship was known for $99.5 \%$ ) of the previous study numbered 223, of whom 19 had moved, 20 could not be contacted at their former address and 8 were too unwell to attend. Of the remaining 176 resident survivors, $88(50.0 \%)$ participated in the follow-up study. Retinal photographs were considered gradable if at least one AMD feature could be allocated a grade for that eye. There were 77 right eyes and 81 left eyes with gradable images, making a total of 158 eyes in 82 subjects for comparison in the final analysis. Of the 82 subjects, $70.7 \%$ were female, and their mean age was 80 years (range 77-90 years) at the initial assessment and 87 years (range 84-97 years) at the subsequent assessment. The mean follow-up period was 6.7 years (SD 0.7 year). In terms of age, sex and vision in the better eye at baseline, the attenders of the follow-up study did not differ significantly from the surviving non-attenders, and attenders were thus considered to be representative of the suvivors as a whole $(p>0.26$ for age and sex, $p=0.10$ for visual acuity $6 / 12$ or better).

\section{Baseline AMD Prevalence}

The prevalence of AMD features scored at the two time points using the Wisconsin grading method is reported elsewhere. ${ }^{15}$

\section{Incidence and Progression of AMD Drusen}

The 7 year incidence of definite drusen of any type was $30.6 \%$ (i.e. of the 36 eyes which were drusen-free at baseline, 11 showed drusen at follow-up). Regression of drusen was observed in $20.0 \%(22 / 110$, Table I). Fig. 1 documents the natural history of maximum drusen size per eye in terms of the change in grade between examinations. Eyes were scored according to the highest-scoring subfield, so the scores document the maximum-sized drusen for each eye as a whole. Eyes with drusen either absent or doubtful (questionable, hard indistinct, or stippling only) on both occasions are separately indicated in Fig. 1. It was found that $39.0 \%$ of eyes scored a higher grade for maximum size at the subsequent examination, whilst $29.5 \%$ showed a lower score. There was no change in score for $31.5 \%$.

The incidence of different types of drusen was determined as follows. Individual subfields were classified according to the type of drusen occupying the largest area of that subfield. Then, assuming a rank-ordering for drusen type of 'hard, soft, reticular', each eye was classified according to the subfield with the 'highest' score along this ranking. By this method the incidence 'by eye' of hard drusen was $7.5 \%$ with soft and reticular drusen having incidence rates of $23.1 \%$ and $19.5 \%$ respectively (Table I gives these incidence rates according to both 'by subfield' and 'by eye' analyses). In order to gain further insight into which changes in drusen type were the predominant changes rather than the 'maximum' change, the 'predominant drusen size' variable was used as a proxy for drusen type (See Method). By this proxy for 'predominant' type, the incidence of hard drusen was $12.0 \%$, whilst that of soft and reticular drusen was approximately $17 \%$ each. By both measures of drusen type, regression rates were generally considerably higher than incidence rates (Table I), a point of interest regarding the fluidity of drusen type over time. The changes in grade over time for both direct and proxy measures of drusen type are illustrated in Fig. 2, each of these demonstrating the rank-ordered progression of increasing grade between initial and subsequent assessments. Separately identified are eyes which had either absent or doubtful drusen on both occasions. (It should be noted that the number of 
Table I. Seven year incidence and regression of drusen

\begin{tabular}{|c|c|c|c|c|c|c|}
\hline \multirow[b]{2}{*}{ Drusen feature } & \multicolumn{2}{|c|}{ Incidence } & \multicolumn{2}{|c|}{ Regression } & \multirow[b]{2}{*}{ Total } & \multirow[b]{2}{*}{ Missing (ungradable) } \\
\hline & $n$ & $\%$. & $n$ & $\%$ & & \\
\hline Definite drusen (by eye) & $11 / 36$ & $30.6 \%$ & $22 / 110$ & $20.0 \%$ & 146 & 12 \\
\hline \multicolumn{7}{|l|}{ Drusen type (by subfield) } \\
\hline Hard & 92/967 & $9.5 \%$ & $161 / 199$ & $80.9 \%$ & 1166 & 256 \\
\hline Soft & $108 / 1031$ & $10.5 \%$ & $88 / 135$ & $65.2 \%$ & 1166 & 256 \\
\hline Reticular & $148 / 1104$ & $13.4 \%$ & $23 / 62$ & $37.1 \%$ & 1166 & 256 \\
\hline \multicolumn{7}{|l|}{ Drusen type (by eye) } \\
\hline Hard & $8 / 106$ & $7.5 \%$ & $34 / 40$ & $85.0 \%$ & 146 & 12 \\
\hline Soft & 24/104 & $23.1 \%$ & $22 / 42$ & $52.4 \%$ & 146 & 12 \\
\hline Reticular & 25/128 & $19.5 \%$ & $5 / 18$ & $27.8 \%$ & 146 & 12 \\
\hline \multicolumn{7}{|c|}{ Drusen type (proxy measure, by eye) } \\
\hline Hard & $13 / 108$ & $12.0 \%$ & $28 / 38$ & $73.7 \%$ & 146 & 12 \\
\hline Soft & $21 / 125$ & $16.8 \%$ & $13 / 21$ & $61.9 \%$ & 146 & 12 \\
\hline Reticular & $21 / 129$ & $16.3 \%$ & $4 / 17$ & $23.5 \%$ & 146 & 12 \\
\hline Confluence & $25 / 89$ & $28.1 \%$ & $16 / 57$ & $28.1 \%$ & 146 & 12 \\
\hline
\end{tabular}

Incidence is the proportion of those free of the feature at baseline which subsequently develop the feature (denominator is those at risk, numerator is those which develop feature). Regression is the proportion of those which have the feature at baseline which subsequently lose the feature (denominator is those with feature, numerator is those which lose the feature).

See text for definition of 'proxy measure' for drusen type.

Incidence and regression rates for drusen type refer strictly to the type under consideration and take no account of whether the score was 'higher' or 'lower' at the contrasted point in time. Thus the incidence of soft drusen could include eyes where there had been no drusen or hard drusen initially, as well as those where reticular drusen had 'disappeared' to leave soft drusen.

Missing drusen data: eyes excluded if image quality did not allow drusen assessment on both occasions.

'absent or doubtful' grade refers to the predominant type of lesion by subfield or by eye and, as such, these numbers are not expected to be exactly the same, and similarly they need not be the same as those in Fig. 1.) An increase in grade occurred in $34.2 \%$ by both measures and a decrease in grade occurred in $18.5 \%$ by the direct measure and $14.4 \%$ by the proxy measure.

Confluence of drusen was defined as the presence of two or more touching drusen, and of 89 eyes with either no drusen or non-confluent drusen at initial assessment, $25(28.1 \%)$ showed confluence at the subsequent assessment. Confluence regressed in exactly the same proportion $(16 / 57$ eyes or $28.1 \%$, see Table I). If the 25 eyes where drusen were either absent or doubtful on both occasions are excluded,

Drusen Size Progression

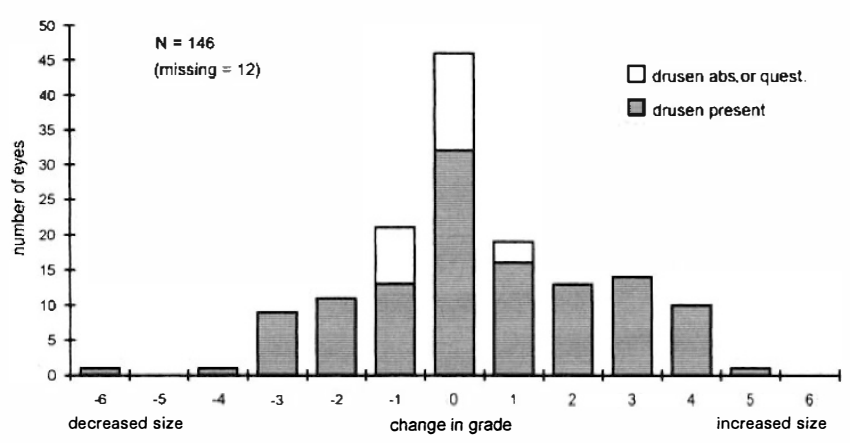

Fig. 1. Change in score between initial and subsequent assessments for maximum drusen size by eye. Where score was either zero or one (drusen absent or doubtful: questionable, hard indistinct or stippling) on both occasions this is indicated by the open extensions to the bars. (Eyes were classified according to highest-scoring subfield; valid scores prior to taking score differences ran between 0 and 6$)$. then within this subgroup of 64 eyes, the 25 with incident confluence constituted $39.1 \%$. Fig. 3 illustrates the change in confluence grade. Separately indicated (on the central bar) are the 25 eyes where drusen were either absent or doubtful on both occasions (grey section) and the further 31 eyes where confluence was absent on both occasions (open section). Overall, $33.6 \%$ of eyes increased their confluence score, $15.1 \%$ decreased their score and $51.4 \%$ showed no change. Those where no

\section{Drusen Type Progression}

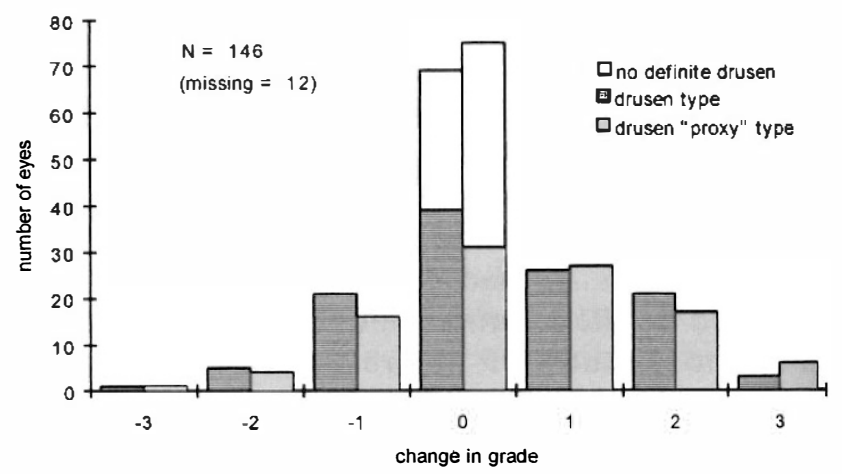

Fig. 2. Change in score between initial and subsequent assessments for drusen type by eye. Two complementary assessments of drusen type are presented. On the left (horizontally hatched bars), the 'highest'-scoring individual subfield was used to classify the eye and on the right (grey bars) a proxy grade referring to the 'predominant' drusen type for the eye as a whole was used. (See text for definition of proxy grade.) The open extensions to the 'zero change' bars indicate eyes where drusen were either absent or doubtful (questionable, hard indistinct or stippled) on both occasions. Valid scores prior to taking score differences were: (1) drusen absent, questionable, hard indistinct or stippled; (2) hard distinct drusen; (3) soft drusen; (4) reticular drusen. 
Drusen Confluence Progression

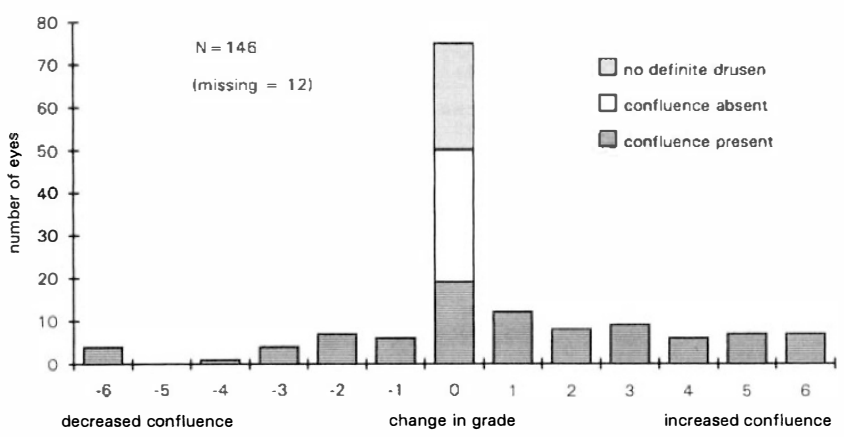

Fig. 3. Change in score between initial and subsequent assessments for drusen confluence by eye. The grey stippled extension on the central bar indicates the 25 eyes in which maximum drusen size scores were absent or doubtful (questionable, stippled or hard indistinct) on both occasions and the open extension indicates the 31 eyes in which drusen were present but non-confluent on both occasions. (Eyes were classified according to highest-scoring subfield and valid scores prior to taking score differences ran between 0 and 6.)

change in confluence occurred included 25 eyes where drusen were either absent or doubtful on both occasions and 31 eyes where confluence was absent on both occasions. If the 25 eyes where drusen were either absent or doubtful on both occasions are excluded, then within this subgroup of eyes with definite drusen on at least one occasion the proportion of eyes which increased in confluence score was $40.5 \%$ and the proportion which decreased in score was $18.2 \%$. In this scoring system there is high sensitivity of the scale to minimal confluence, as the lowest confluence score includes a single touching drusen pair. At the opposite end of the scale the highest confluence score is for reticular drusen, and the observed progression partly reflects the incidence of reticular drusen over the 7 years (Table I).

From the individual subfields, the percentage area occupied by drusen was calculated for each eye as a whole (all subfields combined), as well as for the area defined by the inner and central subfields. ${ }^{17}$ The average increase in percentage area covered by drusen for the eye as a whole was $6.6 \%$, and the equivalent percentage increase for the combined central and inner subfields was $7.5 \%$. A histogram of the change in percentage area of drusen for all subfields combined is shown in Fig. 4. The arithmetic differences in percentage area were plotted in the histogram on a geometric scale in order to illustrate both small and large changes. The 25 eyes with maximum drusen scores which were either absent or doubtful on both occasions are indicated by the open section of the central bar. Large changes occurred in a number of eyes, with a drusen area increase of $40 \%$ or more occurring in 14 eyes. In the subgroup of 121 eyes where definite drusen were present on at least one occasion (Fig. 4, hatched sections), an increase in
Drusen \% Area Progression

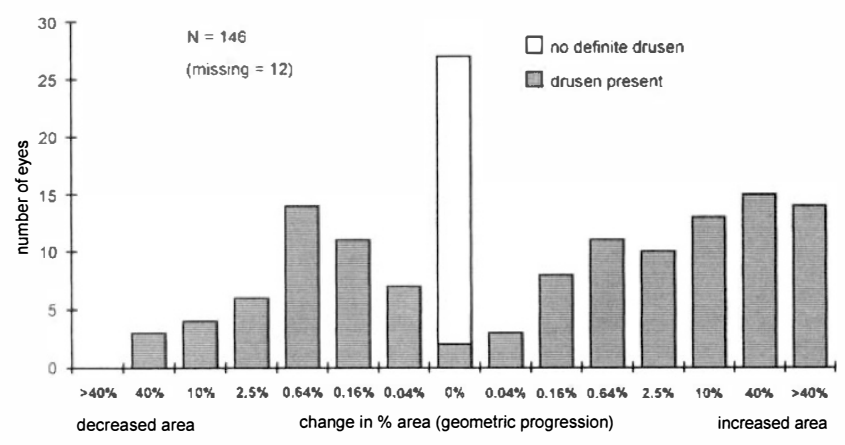

Fig. 4. Change in overall percentage area of drusen between initial and subsequent assessments by eye (all subfields combined). Note that on the graph the percentage area progression proceeds on a geometric scale to highlight small changes in area as well as large changes. Column labels indicate the frequency distribution bins such that from zero outwards each bin label indicates absolute areas larger than the previous label and smaller than or equal to the current label. (The open extension to the central bar indicates eyes where maximum drusen size scores were either absent doubtful on both occasions.)

area of greater than $0.64 \%$ was observed in $43.0 \%$ and an equivalent decrease observed in $10.7 \%$.

\section{Pigmentary Disturbance}

When retinal pigment epithelium (RPE) degeneration was scored by subfield, the incidence was found to be $43.0 \%$ (401/932 subfields). In only $10.5 \%$ (38/362) of subfields was established RPE degeneration scored as having regressed. When the highest subfield score of each eye was used to denote its RPE status, the 7 year incidence of RPE degeneration was $54.5 \%$ (42/77 eyes), while $8.8 \%$ (7/80) of the eyes with RPE degeneration initially were scored as

RPE degeneration \% Area Progression

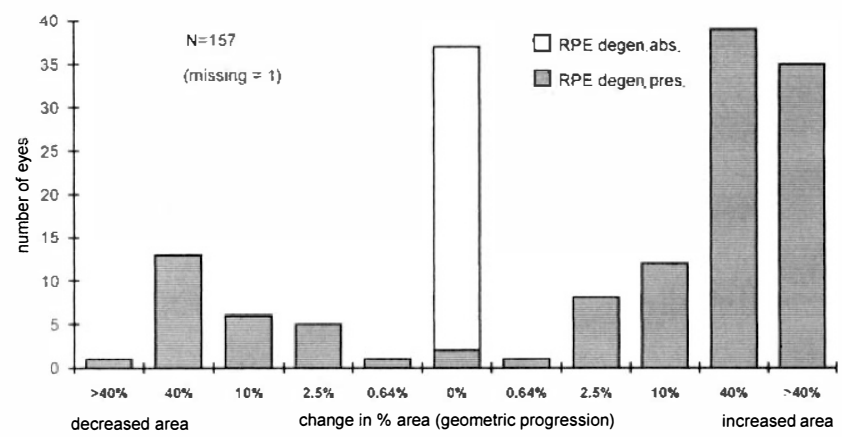

Fig. 5. Change in overall percentage area of RPE degeneration between initial and subsequent assessments by eye (all subfields combined). Note that on the graph, $R P E$ percentage area progression proceeds on a geometric scale to highlight small changes in area as well as large changes. Column labels indicate the frequency distribution bins such that from zero outwards each bin label indicates absolute areas larger than previous label and smaller than or equal to the current label. (The open extension to the central bar indicates eyes where RPE degeneration was either absent or questionable on both occasions.) 


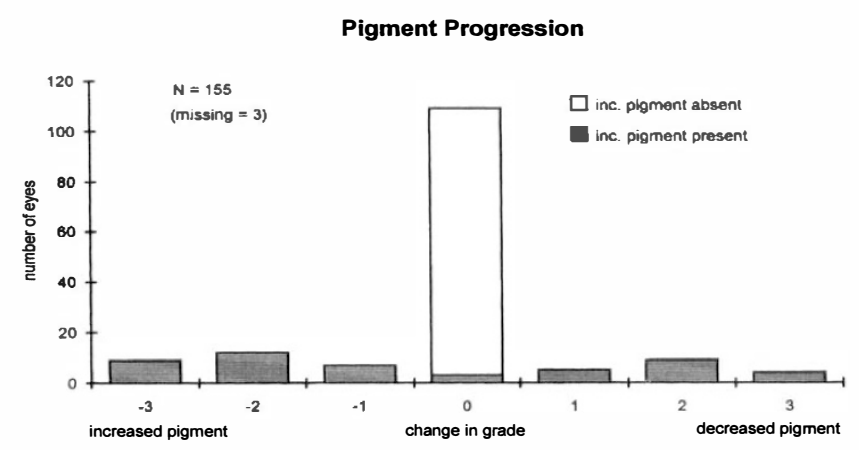

Fig. 6. Change in score between initial and subsequent assessments for increased pigmentation by eye. The open extension to the central bar indicates eyes in which increased pigmentation was absent on both occasions. (Eyes were classified according to highest-scoring subfield; valid scores prior to taking score differences ran between 0 and 3.)

questionable or zero at the subsequent examination (regression). RPE degeneration, when present, was scored according to the percentage area of each affected subfield. From these subfield scores a 'percentage area affected' was calculated for the eye as a whole (all subfields combined) in a manner similar to that used for drusen area. Fig. 5 illustrates the change in percentage area for RPE degeneration. In the subgroup of 120 eyes where definite RPE degeneration was present on at least one occasion (Fig. 5, hatched sections), an increase in area of greater than $2.5 \%$ was observed in $71.7 \%$ and an equivalent decrease observed in $16.7 \%$.

The change in pigment clumping due to AMD was analysed both by subfield and by eye. When increased pigmentation was present at initial examination, there was a striking loss of this pigment at subsequent examination, this regression being observed in $67.0 \%$ of subfields $(59 / 88)$ and $64.7 \%$ of eyes $(22 / 34)$. The regression contrasted with the incidence of this feature over the 7 year period of $3.7 \%$ for subfields $(45 / 1212)$ and $11.6 \%$ for eyes $(14 / 121)$. The histogram of change in pigment grade (Fig. 6) does not, however, provide insight into this apparent regression of increased pigmentation over time. In order to clarify this point two subgroups were analysed. All eyes with pigmentation in the central and inner subfields were considered, exclud-

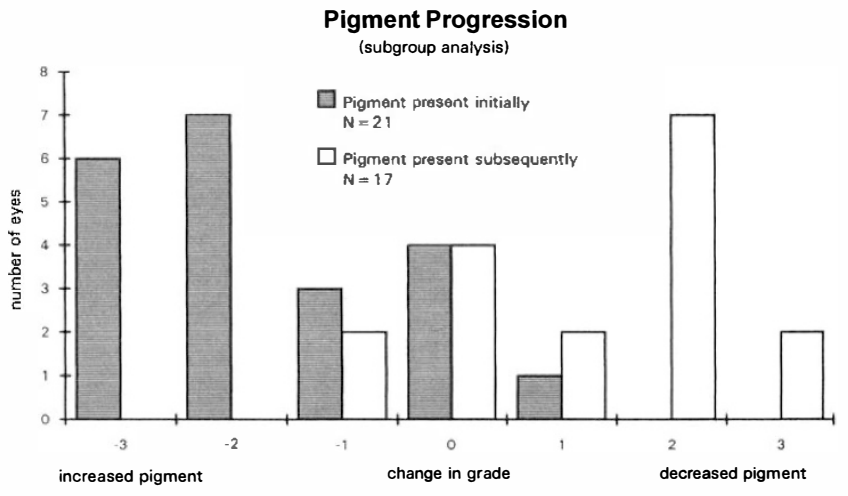

Fig. 7. Subgroup analysis of change in score between initial and subsequent assessments for increased pigment by eye (central and inner subfields only). The two separate analyses show change over time for subgroups, with pigment being present either initially or subsequently to illustrate the transient nature of increased pigment in AMD. (Eyes with photographs of poor quality on either occasion were excluded.)

ing those where either initial or subsequent photographic quality was graded as poor. Fig. 7 separately demonstrates these changes in pigment scores for eyes where increased pigment was present either initially or subsequently. From these histograms, it is apparent that eyes with increased pigment at initial examination lost pigment, whilst those scoring increased pigment subsequently were largely free of pigment initially. These observations, combined with the high rate of regression of pigment noted above, imply that increased pigmentation is a transient event in AMD.

\section{Exudative AMD}

The 7 year incidence of both subretinal scarring/ fibrin deposition and subretinal haemorrhage was $1.3 \%$, with no scars/fibrin disappearing. A single subretinal haemorrhage was present initially. This haemorrhage was due to a macroaneurysm and was not associated with subretinal neovascularisation. At the subsequent examination this haemorrhage had disappeared (Table II).

Table II. Seven year incidence and regression of other AMD features

\begin{tabular}{|c|c|c|c|c|c|c|}
\hline \multirow[b]{2}{*}{ Feature } & \multicolumn{2}{|c|}{ Incidence } & \multicolumn{2}{|c|}{ Regression } & \multirow[b]{2}{*}{ Total } & \multirow[b]{2}{*}{ Missing } \\
\hline & $n$ & $\%$ & $n$ & $\%$ & & \\
\hline RPE by subfield & $401 / 932$ & $43.0 \%$ & $38 / 362$ & $10.5 \%$ & 1294 & 128 \\
\hline RPE by eye & $42 / 77$ & $54.5 \%$ & $7 / 80$ & $8.8 \%$ & 157 & 1 \\
\hline Increased pigmentation by subfield & $45 / 1212$ & $3.7 \%$ & $59 / 88$ & $67.0 \%$ & 1300 & 122 \\
\hline Increased pigmentation by eye & $14 / 121$ & $11.6 \%$ & $22 / 34$ & $64.7 \%$ & 155 & 3 \\
\hline Subretinal haemorrhage & $2 / 156$ & $1.3 \%$ & $1 / 1^{\mathrm{a}}$ & $100 \%$ & 156 & 2 \\
\hline Subretinal scar/fibrin deposition & $2 / 155$ & $1.3 \%$ & $0 / 3$ & $0 \%$ & 158 & 0 \\
\hline Geographic atrophy & $2 / 154$ & $1.3 \%$ & $0 / 3$ & $0 \%$ & 157 & 1 \\
\hline
\end{tabular}

Analysis is 'by eye' unless otherwise indicaed; Total and Missing refer to number of eyes for which data were available on both occasions. RPE, retinal pigment epithelial degeneration.

a This haemorrhage was due to a 'macro-aneurysm' and not AMD. 
Table III. Best corrected Snellen visual acuity by eye at initial and subsequent assessments: $n=147\left(+11^{\mathrm{a}}\right)$

\begin{tabular}{|c|c|c|c|c|c|c|c|c|c|c|}
\hline \multirow[b]{2}{*}{ Initial } & \multicolumn{10}{|c|}{ Subsequent } \\
\hline & $6 / 6$ & $6 / 9$ & $6 / 12$ & $6 / 18$ & $6 / 24$ & $6 / 36$ & $6 / 60$ & $\mathrm{CF}$ & HM & PL \\
\hline$\overline{\mathrm{PL}}$ & 0 & 0 & 0 & 0 & 0 & 0 & 0 & 1 & 1 & 0 \\
\hline HM & 0 & 0 & 0 & 0 & 0 & 0 & 0 & 1 & 1 & 0 \\
\hline CF & 0 & 0 & 0 & 0 & 0 & 0 & 1 & $4(1)$ & 1 & 1 \\
\hline $6 / 60$ & 0 & $0(1)$ & 0 & 0 & 0 & 0 & 2 & 1 & 0 & 0 \\
\hline $6 / 36$ & $0(1)$ & 0 & 1 & 0 & 1 & 0 & 2 & 1 & 0 & 0 \\
\hline $6 / 24$ & 0 & $0(2)$ & 0 & 1 & 3 & 0 & 1 & 1 & 1 & 0 \\
\hline $6 / 18$ & 0 & 1 (1) & $3(1)$ & 3 & 2 & 1 & 1 & 2 & 0 & 0 \\
\hline $6 / 12$ & 1 & 6 & 4 & 5 & 5 & 0 & 0 & 2 & $0(1)$ & 0 \\
\hline $6 / 9$ & $4(1)$ & 25 & 15 & 8 & 8 & 3 & 0 & $1(1)$ & 1 & 0 \\
\hline $6 / 6$ & 2 & 7 & 4 & 2 & $2(1)$ & 0 & 0 & 0 & 2 & 1 \\
\hline
\end{tabular}

${ }^{a}$ Eyes which underwent cataract extraction during follow-up are separately indicated in brackets.

\section{Geographic atrophy}

Geographic atrophy had a similar incidence to exudative AMD (1.3\% or $2 / 154$ eyes) and, not surprisingly, did not disappear in the 3 eyes which showed this feature initially (Table II).

\section{Visual Acuity}

Table III compares the best corrected Snellen visual acuities by eye at the initial and subsequent assessments (11 eyes which underwent cataract extraction between initial and subsequent assessments are indicated separately). Excluding eyes which underwent cataract surgery during the follow-up period, acuity remained within 2 Snellen lines for 119 eyes $(81.0 \%), 27$ eyes $(22.7 \%)$ lost 3 or more Snellen lines, and 1 eye improved by 3 lines. Including the 11 eyes which underwent cataract surgery during follow-up, the incidence of loss of vision to $6 / 18$ or worse (in eyes which had initially achieved $6 / 12$ or better) was $27.2 \%$, and the converse incidence of visual improvement in eyes which had initially had vision of $6 / 18$ or worse was $7.0 \%$. This apparent visual improvement was less evident $(3.4 \%)$ when eyes undergoing cataract surgery were excluded. Overall, the incidence of blindness $(6 / 60$ or worse) was $11.4 \%$ and vision was restored by cataract surgery to one initially blind eye.

\section{Summary of AMD Changes Between Initial and Subsequent Assessments}

Clinically relevant summary measures of AMD severity were defined from the detailed grades (see Method). Table IV indicates the summarised AMD status by eye at initial and subsequent assessments. The first section in this table provides information on all 158 eyes, with the 11 eyes undergoing cataract extraction during the follow-up period separately identified in brackets. Of the 158 eyes, 78 (49.4\%) had the same summary status on each occasion, in 63 eyes $(39.9 \%)$ the summary AMD status progressed, and 17 eyes $(10.8 \%)$ showed regression. By this summary AMD measure, it is apparent that far more eyes progressed than regressed.

Table IV. Summary of AMD status overall and according to change in visual acuity between initial and subsequent assessments

\begin{tabular}{|c|c|c|c|}
\hline \multirow[b]{2}{*}{ Subsequent } & \multicolumn{3}{|c|}{ Initial } \\
\hline & None & Minor AMD & Major AMD \\
\hline \multicolumn{4}{|c|}{ All eyes, $n=147(+11)$} \\
\hline None & 11 & 10 & 2 \\
\hline Minor AMD & $12(1)$ & 34 & 5 \\
\hline Major AMD & 3 & $37(10)$ & 33 \\
\hline \multicolumn{4}{|c|}{3 or more Snellen lines lost, $n=27(+3)$} \\
\hline None & 0 & 3 & 0 \\
\hline Major AMD & 0 & $6(3)$ & 11 \\
\hline \multicolumn{4}{|c|}{$6 / 6$ or better to $6 / 9$ or worse, $n=18(+1)$} \\
\hline None & 1 & 1 & 0 \\
\hline Minor AMD & 1 & 4 & 0 \\
\hline Major AMD & 0 & $5(1)$ & 6 \\
\hline \multicolumn{4}{|c|}{$6 / 36$ or better to $6 / 60$ or worse, $n=16(+2)$} \\
\hline None & 2 & $\cdot 1$ & 0 \\
\hline Minor AMD & 0 & 1 & 0 \\
\hline
\end{tabular}

Analysis 'by eye'; 11 eyes which underwent cataract extraction during the follow-up period are separately indicated in brackets. None, AMD features either absent or questionable in the worst-affected subfield.

Minor $A M D$, definite drusen which were not reticular; with confluence $<500 \mu \mathrm{m}$ and overall area $<10 \%$ of all subfields combined (entire grid area); RPE degeneration with overall area involving $<25 \%$ of all subfields combined; and increased pigmentation.

Major $A M D$, any reticular drusen, drusen with confluence $\geqslant 500 \mu \mathrm{m}$ and/or drusen area $\geqslant 10 \%$ of all subfields combined; RPE degeneration occupying $\geqslant 25 \%$ of all subfields combined; exudative AMD (subretinal haemorrhage or scar/fibrin in any subfield); geographic atrophy (any subfield). 


\section{AMD Changes in Relation to Visual Loss}

The second section in Table IV represents the summary AMD status in the subgroup of 30 eyes in which visual acuity decreased by 3 or more Snellen lines between initial and subsequent examinations. In 10 of these eyes (33\%) AMD status progressed while in only 3 eyes $(10 \%)$ was regression observed. The third section in Table IV represents the subgroup of 19 eyes in which visual acuity was initially normal (6/6 or better), and decreased to $6 / 9$ or worse. AMD status progressed in 7 of these eyes $(37 \%)$, with only 1 eye $(5 \%)$ showing regression. The final section in Table IV indicates AMD status in the 18 eyes which crossed the blindness threshold from $6 / 36$ or better initially to $6 / 60$ or worse subsequently. Five of these eyes $(28 \%)$ showed progression of AMD while only 1 eye $(6 \%)$ showed regression. These results confirm in general that eyes which lost Snellen acuity during follow-up also demonstrated a progression of AMD over the 7 year period.

\section{DISCUSSION}

The population-based incidence and natural history data presented in this study are unique. The very elderly age structure of the population and the long follow-up period make the observations on this geographically representative population particularly relevant to the study of AMD.

At the baseline examination the participants were demographically similar to the non-participants. ${ }^{16}$ At the follow-up examination the participants were again demographically similar to the non-participants, although there may have been an underrepresentation of visual morbidity. ${ }^{15}$ This could have occurred if visually disabled individuals had been less likely to participate in the original eye study, or if individuals whose vision had deteriorated during the follow-up period had been less likely to participate in the follow-up assessment. ${ }^{12}$ Thus it may be that these data represent minimum figures for the morbidity of AMD in an elderly population, which is of great importance when considering the expected demographic population shifts and changing health care requirements of the coming decades.

The measure of incidence used in this analysis was the percentage of eyes initially free of a feature that went on to develop that feature during the period of follow-up. For features that are irreversible and unrelated to mortality or other loss to follow-up, this is a measure of risk, which for rare conditions will closely approximate the cumulative incidence. However, some caution needs to be exercised in interpreting these results or in comparing them with results from other studies, particularly when the feature may be reversible or when the condition is not rare.
The AMD features with the highest overall incidence in this study are RPE degeneration and drusen of any type. The clinical appearances of both features can at times be subtle, and their visibility may be influenced by lens opacities. These opacities tend to increase, of course, with time. If the visibility of the fundus is deteriorating with age, then the true incidence of subtle lesions may have been even higher than the data suggest, and the true regression lower.

A limitation of the Wisconsin method of classification and grading of individual retinal subfields is that it is not possible to state precisely the 'by eye' incidence of a complicated variable such as drusen type. As discussed elsewhere, the true incidence of hard and soft drusen are not directly measured in this classification system. ${ }^{15}$ Drusen type for the eye as a whole must therefore be approximated from amalgamated subfield data on drusen type, or (as we have done) from data on the predominant size of drusen in the grid area as a whole. Nevertheless, our data do show that drusen come and go, that the predominant drusen type in an eye changes over time, and that this change tends to be towards larger-sized and reticular drusen, with confluence increasing.

These observations on drusen progression are compatible with the prevalence studies of Klein et al., ${ }^{17}$ who note that the prevalence of small hard drusen decreases with age, whereas the prevalence of larger drusen increases with age, as do drusen confluence and area. In a 5 year follow-up study, Bressler et al..$^{5}$ also noted regression of large drusen, and although their population was exclusively male and much younger than that of the present study, similarities are evident. Other workers have likewise reported similar findings. ${ }^{18-22}$ Histological studies by Sarks et al. $^{23}$ support these in vivo observations by demonstrating intermediate histological forms which imply metamorphosis of hard drusen to soft drusen. Sarks, however, found no extracellular material which corresponded with the clinical appearance of reticular drusen, and attributed reticular drusen to choroidal vascular insufficiency.

The temporal variability of macular pigmentary disturbances is remarkable in that it demonstrates the disappearance of clumps of increased pigmentation. Following the analysis of the initial and subsequent observations (masked), the relevant photographs were reviewed to check and confirm that the apparent disappearance of pigment was not an artefact produced by changes in visibility. Side-by-side review confirmed that pigment was often linearly disposed at the interface between more healthy-looking and less healthy-looking RPE, and when increased pigmentation resolved, it left a discrete patch of RPE degeneration (though not geographic atrophy, as defined by the Wisconsin grading protocol). A junc- 
tional zone of severe RPE degeneration adjacent to geographic atrophy is described in the histological studies of Sarks et $a l^{24}$ This zone, thought to be of relevance in the evolution of geographic atrophy, contained large RPE cells 'densely packed with pigment granules' which were shed into the subretinal space, in which pigment-laden giant cells were also present. From this it was concluded that the dark pigmented border was due to pigment which had been shed into the subretinal space, and we suggest that this may have been the histological equivalent of the linear pigmentation seen in our study. Variable nomenclature rather than differences in pathology may account for the apparent inconsistencies between subsequent development of RPE degeneration and geographic atrophy in the two studies. In addition to the inference of a receding zone of hyperpigmentation in Sarks' study, ${ }^{24}$ and the disappearance of salt and pepper pigmentation noted by Schatz and McDonald, ${ }^{25}$ Bressler et $a l^{5}$ noted a high rate of disappearance of focal hyperpigmentation in their follow-up study. Our findings, together with the observations of other authors, suggest that the complete disappearance of whole clumps of pigment is not uncommon, i.e. that increased pigmentation can be a transient feature of AMD. This may be of relevance to the finding by several workers that focal pigment clumping is associated with an increased risk of subretinal neovascular membrane formation. ${ }^{19,26,27}$

RPE degeneration, regardless of pigment clumping, is clearly shown to have a high incidence over the 7 years of this study. Schatz and McDonald ${ }^{25}$ in their follow-up study of individuals with geographic atrophy comment that geographic atrophy follows RPE degeneration. In our sample, despite the high prevalence and incidence of RPE degeneration, the incidence of geographic atrophy was low. Once again, the explanation for this may be the variable definitions used by different authors to distinguish severe RPE degeneration from geographic atrophy.

The incidence of exudative AMD in this populationbased sample is very low in comparison to that reported by Smiddy and Fine ${ }^{26}$ of $9.6 \%$ over 5 years. Their data, however, refer to a highly selected sample of patients with bilateral drusen referred to a dedicated retinal centre, and as such would be expected to have relatively more severe AMD features than the general population. Over a 5 year follow-up Bressler et al. ${ }^{5}$ found a single occurrence of neovascular AMD in the 50 participants aged over 70 years. This $2 \%$ incidence per person would be compatible with our rate per eye of $1.3 \%$ over 7 years. For reasons already discussed, the present report may represent an underestimate of the true incidence of severe AMD in the elderly population at large; however, the number of affected eyes is small and caution should be exercised when interpreting these rates.
From the perspective of the elderly individual, the incidence of visual loss in AMD and the prognosis for the maintenance of vision are the most relevant parameters. In our study, of the 27 eyes which were disease free initially, 16 (59\%) developed one or more features of AMD over the 7 years (Table IV, first section). In only one of these, however, was there an associated drop in acuity of $\geqslant 3$ Snellen lines. Similarly, of those 47 eyes which progressed from minor AMD to major AMD, only 9 eyes (19\%) lost $>3$ Snellen lines, and only 5 of these 47 eyes $(11 \%)$ became legally blind (6/60 or worse). Of the 18 eyes which became legally blind during the study, half $(9 / 18)$ did have features of major AMD at initial assessment (Table IV, final section). Thus, although the overall incidence of blindness in this population was 18 of 158 eyes $(11.4 \%)$ over 7 years, the incidence of blinding disease in the subgroup of those 40 eyes showing features of major AMD at baseline examination was more than double the overall rate, i.e. 9 of $40(23 \%)$. As detailed elsewhere ${ }^{15}$ the prevalence of major AMD in this population at subsequent assessment (mean age 87 years) was over $50 \%$. This study therefore suggests that as the number of people living to over 85 years continues to increase, the number of individuals with severe visual impairment due to AMD is likely to rise considerably, despite an apparently low incidence of both exudative AMD and geographic atrophy.

\section{CONCLUSION}

Our study is the first population-based long-term incidence study of AMD in an elderly population. Incidence and regression rates for various AMD lesions have been described over a 7 year period, and information on change in grade (disease severity) over the period have been presented. These population-based data provide fresh insight into the natural history of AMD from the perspective of disease incidence and progression over time.

The authors wish to thank the Latham House Medical Practitioners for allowing us to examine their patients, Professor Michael Clarke and the Department of Epidemiology and Public Health of the University of Leicester for population and survivorship information, Hugh Harris for photographic assistance, and Kath Robinson and Rachel Gowing for clerical assistance. This study was supported in part by the Anne Allerton fund and by the National Eye Research Council.

Key words: Age-related macular degeneration, Incidence, Natural history.

\section{REFERENCES}

1. Green WR, McDonnell PJ, Yeo JH. Pathological features of senile macular degeneration. Ophthalmology 1985;92:615-27. 
2. Vinding T. Age-related macular degeneration: macular changes, prevalence and sex ratio. An epidemiological study of 100 aged individuals. Acta Ophthalmol (Copenh) 1989;67:609-16.

3. Evans JR, Wormald RPL. Epidemiological function of BD8 certification. Eye 1993;7:172-9.

4. Cheraskin E. Macular degeneration: how big is the problem? J Natl Med Assoc 1992;84:873-6.

5. Bressler NM, Munoz B, Maguire MG, et al. Five year incidence and disappearance of drusen and retinal pigment epithelial abnormalities (Waterman study). Arch Ophthalmol 1995;113:301-8.

6. Gregor Z, Bird AC, Chisholm IH. Senile disciform macular degeneration in the second eye. $\mathrm{Br} J$ Ophthalmol 1977;61:141-7.

7. Bressler NM, Bressler SB, Seddon JM, Gragoudas ES, Jacobson LP. Drusen characteristics in patients with exudative versus non-exudative age-related macular degeneration. Retina 1988;8:109-14.

8. Bressler NM, Bressler SB, Fine SL. Age-related macular degeneration. Surv Ophthalmol 1988;32: 375-413.

9. Klein R, Davis MD, Magli YL, Segal P, Klein BE, Hubbard L. The Wisconsin age-related maculopathy grading system. Ophthalmology 1991;98:1128-34.

10. Sparrow JM, Dickinson AJ, Duke AM. The Wisconsin Age-related Macular Degeneration Grading System: performance in an independent centre. Ophthalmic Epidemiol 1997;4:49-55.

11. Leibowitz HM, Krueger DE, Maunder LR, et al. The Framingham Eye Study Monograph. Surv Ophthalmol 1980;24(Suppl):335-610.

12. Martinez GS, Campbell AJ, Reinken J, Allan BC. Prevalence of ocular disease in a population study of subjects 65 years old and older. Am J Ophthalmol 1982;94:181-9.

13. Lui IY, White L, LaCroix AZ. The association of agerelated macular degeneration and lens opacities in the aged. Am J Public Health 1989;79:765-9.

14. Clarke M, Clarke SM, Odell A, Jagger C. The elderly at home: health and social status. Health Trends 1984;16:3-7.
15. Dickinson AJ, Sparrow JM, Duke AM, Thompson JR, Gibson JM, Rosenthal AR. Prevalence of age-related macular degeneration at two time points in an elderly British population. Eye 1997;11:301-14.

16. Gibson JM, Rosenthal AR, Lavery J. A study of the prevalence of eye disease in the elderly in a community. Trans Ophthalmol Soc UK 1985;104:196-203.

17. Klein R, Klein BEK, Linton KLP. Prevalence of agerelated maculopathy: the Beaver Dam Eye Study. Ophthalmology 1992;99:933-43.

18. Sarks SH. Ageing and degeneration in the macular region: a clinico-pathological study. $\mathrm{Br} \mathrm{J}$ Ophthalmol 1976;60:324-41.

19. Sarks SH. Drusen and their relationship to senile macular degeneration. Aust J Ophthalmol 1980;8: 117-30.

20. Coffey AJH, Brownstein S. The prevalence of macular drusen in postmortem eyes. Am J Ophthalmol 1986; 102:164-71.

21. Lewis H, Straatsma BR, Foos RY. The prevalence of macular drusen in postmortem eyes. Am J Ophthalmol 1986;102:801-2.

22. van der Schaft TL, Mooy CM, de Bruijn WC, Oron FG, Mulder PG, de Jong PT. Histological features of the early stages of age-related macular degeneration: a statistical analysis. Ophthalmology 1992;99:278-86.

23. Sarks JP, Sarks SH, Killingsworth MC. Evolution of soft drusen in age-related macular degeneration. Eye 1994;8:269-83.

24. Sarks JP, Sarks SH, Killingsworth MC. Evolution of geographic atrophy of the retinal pigment epithelium. Eye 1988;2:552-77.

25. Schatz H, McDonald HR. Atrophic macular degeneration: rate of spread of geographic atrophy and visual loss. Ophthalmology 1989;96:1541-51.

26. Smiddy WE, Fine SL. Prognosis of patients with bilateral macular drusen. Ophthalmology 1984;91: 271-7.

27. Bressler SB, Maguire MG, Bressler NM, Fine SL. Relationship of drusen and abnormalities of the retinal pigment epithelium to the prognosis of neovascular macular degeneration. The Macular Photocoagulation Study Group. Arch Ophthalmol 1990;108:1442-7. 